\title{
Possibilidades do uso da terra de diatomáceas na composição de materiais odontológicos: revisão integrativa
}

Possibilities of the use of diatomaceous earth in the composition of dental materials: integrative review

Posibilidades del uso de diatomace tierra en la composición de materiales dentales: revisión integrativa

ORCID: https://orcid.org/0000-0002-2911-3549 Universidade do Estado do Rio Grande do Norte, Brasil E-mail: t_alitapinto@outlook.com

Heloísa Pereira de Medeiros

ORCID: https://orcid.org/0000-0001-5769-4988 Universidade do Estado do Rio Grande do Norte, Brasil E-mail: heloisap.medeiros@gmail.com

Hiully Karydja Câmara Oliveira

ORCID: https://orcid.org/0000-0001-6845-4592

Universidade do Estado do Rio Grande do Norte, Brasil

E-mail: hiully_karydja1995@hotmail.com

\begin{abstract}
Resumo
Introdução: Dentre os avanços na Engenharia de materiais com fins odontológicos está a inclusão de novas e/ou subutlizadas matérias primas para a obtenção de melhores características físicas e, consequentemente, longevidade clínica dos produtos, uma destas é a terra de diatomáceas ou diatomita. Objetivo: Compilar as principais possibilidades de uso da diatomita na composição de materiais odontológicos. Metodologia: Realizou-se revisão integrativa, com busca de alta sensibilidade nas bases de dados: Medline via PubMed, Cochrane Wiley e Embase, e no portal: BVS, utilizando os descritores "Diatomaceous Earth" e "Dental Materials" na língua inglesa, assim como o sinônimo "Infusorial Earth" relacionados com operador booleano "AND". A triagem dos artigos encontrados foi realizada por meio do aplicativo Rayyan ${ }^{\circledR}$. Resultados: Foram encontrados 51 estudos. Após leitura do título, resumo e artigo na íntegra, apenas quatro estudos foram passíveis de inclusão. Não houve trabalhos que discorressem, especificamente, sobre o uso da terra de diatomácea em materiais odontológicos, porém, esses estudos apresentam a análise de materiais odontológicos e/ou biomateriais que possuem a diatomita. Os trabalhos inclusos foram publicados entre 2008 e 2014, sendo todos da língua inglesa. Conclusão: A terra de diatomáceas pode ser utilizada em diferentes tipos de materiais odontológicos, principalmente, com o objetivo de garantir melhores propriedades físicas, porém, ainda se faz necessária a realização de novas pesquisas para analisar suas possibilidades industriais.
\end{abstract}

Palavras-chave: Terra de diatomáceas; Materiais dentários; Odontologia.

\begin{abstract}
Introduction: Among the advances in materials engineering for dental purposes is the inclusion of new and/or underutilized raw materials to obtain the best physical characteristics and, consequently, the clinical longevity of the products, one of these being diatomaceous earth or diatomite. Objective: Compile the main possibilities of use of diatomite in the composition of dental materials. Methodology: An integrative review was carried out, through a high sensitivity search in the databases: Medline via PubMed, Cochrane Wiley and Embase, and in the portal: BVS, using the descriptors "Diatomaceous Earth" and "Dental Materials" in English, as well as the synonym "Infusorial Earth" related with the Boolean Operator "AND". The selection of the articles found was performed using the Rayyan ${ }^{\circledR}$ application. Results: There were 51 studies found in the search. After reading the title, abstract and full article, four studies were included. There were no studies that specifically discuss the use of diatomaceous earth in different dental materials, but these studies presented the analysis of dental materials and/or biomaterials that had diatomite. The
\end{abstract}


included papers were published between 2008 and 2014, all in English. Conclusion: Diatomaceous earth can be used in different types of dental materials, mainly to ensure better physical and mechanical properties, but more research is still needed to analyze its possibilities completely.

Keywords: Diatomaceous earth; Dental materials; Dentistry.

\section{Resumen}

Introducción: Entre los avances en la ingeniería de materiales con fines odontológicos se encuentra la inclusión de materias primas nuevas y / o subutilizadas para obtener mejores características físicas y, en consecuencia, longevidad clínica de los productos, siendo una de ellas la tierra de diatomeas o la diatomita. Objetivo: recopilar las principales posibilidades de uso de diatomita en la composición de materiales dentales. Metodología: Se realizó una revisión integradora, con búsqueda de alta sensibilidad en las bases de datos: Medline vía PubMed, Cochrane Wiley y Embase, y en el portal: BVS, utilizando los descriptores "Diatomaceous Earth" y "Dental Materials" en inglés, así como el sinónimo "Infusorial Earth" relacionado con el operador booleano "Y". El cribado de los artículos encontrados se realizó mediante la aplicación Rayyan®. Resultados: Se encontraron 51 estudios. Después de leer el título, el resumen y el artículo completo, solo cuatro estudios fueron elegibles para su inclusión. No hubo estudios que discutieran específicamente el uso de tierra de diatomeas en materiales dentales, sin embargo, estos estudios presentan el análisis de materiales dentales y / o biomateriales que contienen diatomita. Los trabajos incluidos fueron publicados entre 2008 y 2014, todos en inglés. Conclusión: La tierra de diatomeas se puede utilizar en diferentes tipos de materiales dentales, principalmente con el objetivo de garantizar mejores propiedades físicas, sin embargo, aún es necesario realizar más investigaciones para analizar sus posibilidades industriales.

Palabras clave: Tierra de diatomeas; Materiales dentales; Odontología.

\section{Introdução}

A Odontologia é uma ciência que acompanha as evoluções tecnológicas na área da saúde, sempre se adaptando com os objetivos principais de garantir o tratamento mais adequado para o paciente e, melhores condições de trabalho para o profissional. Associados a isso, estão os avanços inerentes aos materiais odontológicos, que propiciam melhores propriedades destes e, consequentemente, maior probabilidade de sucesso do procedimento e longevidade clínica (Chain, 2013).

Dentre os avanços na Engenharia de materiais com fins odontológicos está a inclusão de novas e/ou subutilizadas matérias primas para a obtenção de melhores características dos produtos. Nesse cenário surgiu o uso de diferentes elementos, como a diatomita. A terra de diatomáceas ou diatomita consiste em exoesqueletos mineralizados de algas unicelulares (diatomáceas) envoltos por uma parede celular de sílica (dióxido de silício), $\mathrm{SiO}_{2}$ (Guiry, 1997) (Elias et al., 2006) (Holmes et al., 2006).

É importante salientar que embora possa ser sintetizada em laboratório, a obtenção de sílica por meio de diatomita é o método mais vantajoso, principalmente, devido a sua grande quantidade na natureza e o alto custo-benefício, visto que o custo é baixo perante as várias possibilidades de uso (Guiry, 1997) (Elias et al., 2006) (Holmes et al., 2006).

Além disso, a diatomita é um silicato que apresenta alta capacidade de absorção, baixa densidade e compatibilidade com os tecidos humanos e metais pesados, como os usados em cerâmicas para próteses dentárias (Losic et al., 2009) (Şan et al., 2009) (Hadjadj-Aoul et al., 2005) (Ediz et al., 2010). Devido a isso, a diatomita apresenta aplicações versáteis dentro da Odontologia.

Sendo assim, as diferentes vantagens do uso da terra diatomácea junto com o constante avanço tecnológico no campo dos materiais odontológicos tornam cruciais pesquisas que permitam um apanhado do uso dessa matéria prima e que possam servir de base para novas pesquisas na área. Nesse contexto, esta pesquisa tem como objetivo compilar as principais possibilidades de uso da diatomita na composição de materiais odontológicos.

\section{Metodologia}

Trata-se de uma revisão integrativa da literatura. Método que visa a síntese sobre documentos científicos disponíveis e, posteriormente, análise e aplicabilidade dos resultados encontrados (Souza et al., 2010). 
A realização deste trabalho seguiu as seis fases preconizadas para realização de uma revisão integrativa - sendo essas: $1^{\mathrm{a}}$ fase: elaboração da pergunta de pesquisa; $2^{\mathrm{a}}$ fase: busca na literatura; $3^{\mathrm{a}}$ fase: coleta dos dados; $4^{\mathrm{a}}$ fase: análise dos estudos

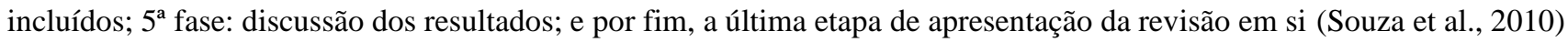

Para tanto a questão norteadora foi: “Quais são as possibilidades para o uso da terra de diatomáceas na composição de materiais odontológicos?". Para respondê-la, foi realizada uma busca nas seguintes bases de dados: Medline via Pubmed, Cochrane Wiley e Embase, assim como no Portal de dados BvS. A busca foi realizada usando os descritores "Diatomaceous Earth" e "Dental Materials" na língua inglesa, assim como o sinônimo "Infusorial Earth" sendo combinados por meio do operador booleano “AND”, conforme o quadro a seguir.

Quadro 1. Estratégia de buscas de acordo com as bases ou portal de dados pesquisados.

\begin{tabular}{|c|l|}
\hline $\begin{array}{c}\text { Bases de Dados } \\
\text { Portal de Dados }\end{array}$ & \multicolumn{1}{|c|}{ Estratégia de busca } \\
\hline $\begin{array}{c}\text { Medline via } \\
\text { PubMed }\end{array}$ & $\begin{array}{l}\text { "Diatomaceous Earth"[Mesh] OR (Diatomaceous Earth) OR (Earth, Diatomaceous) OR (Infusorial } \\
\text { Earth) OR (Earth, Infusorial) OR (Kieselguhr) AND "Dental Materials"[Mesh] OR (Dental } \\
\text { Materials) OR (Materials, Dental) OR (Dental Material) OR (Material, Dental) }\end{array}$ \\
\hline Cochrane Wiley & $\begin{array}{l}\text { (Diatomaceous Earth) OR (Diatomaceous Earth) OR (Earth, Diatomaceous) OR (Infusorial Earth) } \\
\text { OR (Earth, Infusorial) OR (Kieselguhr) AND (Dental Materials) OR (Dental Materials) OR } \\
\text { (Materials, Dental) OR (Dental Material) OR (Material, Dental) }\end{array}$ \\
\hline Embase via Elsevier & $\begin{array}{l}\text { 'infusorial earth'/exp OR (infusorial earth) OR (celite) OR (diatomaceous earth) OR (diatomite) } \\
\text { OR (fossil flour) OR (kieselguhr) OR (siliceous earth) AND 'dental material'/exp OR (dental } \\
\text { material) OR (dental materials) OR (material, dental) }\end{array}$ \\
\hline \multicolumn{1}{|c|}{ BVS: "Terra de Diatomáceas" OR (Terra de Diatomáceas) OR (Diatomaceous Earth) OR (Tierra } \\
de Diatomeas) OR (Kieselguhr) OR (Terra de infusórios) OR (Terras de Diatomáceas) OR \\
D01.578.750.300\$ OR D01.650.550.825.400\$ OR D01.837.725.400\$ AND MH: "Materiais \\
Dentários" OR (Materiais Dentários) OR (Dental Materials) OR (Materiales Dentales) OR \\
D25.339\$ OR D27.720.102.339\$ OR J01.637.051.339\$
\end{tabular}

Fonte: Autores (2021).

Foram incluídos trabalhos que discorressem sobre o uso da terra de diatomáceas na composição de materiais odontológicos e devido ao número limitado de trabalhos não houve critérios de exclusão quanto ao tipo de estudos.

A pesquisa e análise dos trabalhos encontrados foram realizadas por dois pesquisadores no mês de abril de 2021, após esse processo foi constatada divergência quanto a inclusão de 09 artigos, porém, um consenso foi alcançado após a avaliação de um terceiro pesquisador. A remoção de duplicatas e a triagem dos artigos foram executadas no programa Rayyan: inteligence systematic review ${ }^{\odot}($ Ouzzani et al., 2016).

O processo de seleção da amostra está exposto no fluxograma abaixo: 
Figura 1. Fluxograma do processo de seleção dos trabalhos.

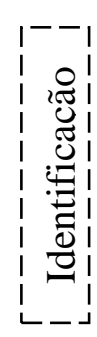

Registros identificados nas bases de dados. $(n=71)$

PubMed: 15; BvS: 12; Embase: 44; Cochrane: 0

Registros removidos antes da triagem:

Registros duplicados $(\mathrm{n}=20)$ avaliados para elegibilidade $(n=13)$

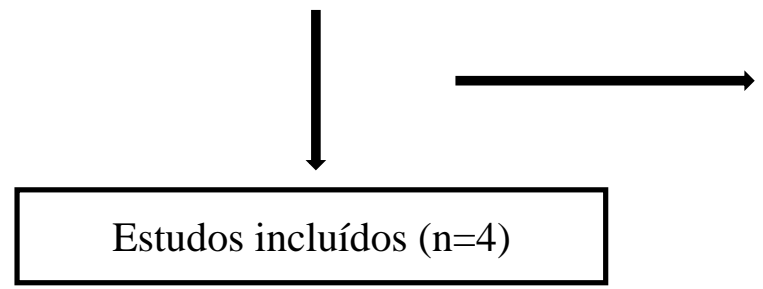

Registros excluídos após leitura de título e resumo $(\mathrm{n}=38)$

Fonte: Autores (2021).

Os principais dados dos registros incluídos foram extraídos e aplicados em uma ficha de análise padrão, previamente elaborada e aplicada para avaliação dos estudos recrutados. Na ficha estão detalhadas as principais informações dos estudos, como título, ano de publicação, autores objetivo, delineamento do estudo e principais resultados.

\section{Resultados}

Após a busca nas bases de dados os artigos incluídos foram analisados e organizados na tabela 1 e o uso da diatomita relatado em cada trabalho na Tabela 2. Foram selecionados quatro artigos, publicados entre os anos 2008 e 2012 , sendo todos escritos na língua inglesa. Não houve trabalhos que discorressem, especificamente, sobre o uso da terra de diatomácea em diferentes materiais odontológicos, porém esses estudos apresentam a análise de materiais odontológicos e/ou biomateriais que possuíam a diatomita em sua composição. Quanto à natureza dos estudos, consistiram em um artigo de opinião de especialistas e 03 três estudos laboratoriais, conforme demonstra a Tabela 1. 
Tabela 1. Descrição dos estudos selecionados.

\begin{tabular}{lcc}
\hline Variáveis & n & \% \\
\hline Ano de publicação & & $25 \%$ \\
2008 & 1 & $25 \%$ \\
2011 & 1 & $25 \%$ \\
2012 & 1 & $25 \%$ \\
2014 & 1 & $100 \%$ \\
Idioma & & \\
Inglês & 4 & $25 \%$ \\
Tipos de estudo & & $75 \%$ \\
Opinião de especialista & 1 & \\
Estudo laboratorial & 3 & \\
\hline
\end{tabular}

Fonte: Autores (2021).

Após a leitura na íntegra dos estudos foi realizada uma compilação e exposição dos principais achados sobre as possibilidades de uso da diatomita na composição de materiais odontológicos por cada autor (Tabela 2).

Tabela 2. Autores e os principais resultados para o uso da diatomita.

\begin{tabular}{ll}
\hline Autores & Resultados da pesquisa/ uso da diatomita \\
\hline Xiaohong Wang; et.al & $\begin{array}{l}\text { A sílica e suas enzimas podem agir induzindo } \\
\text { anabolicamente a síntese de hidroxiapatita (HA) e } \\
\text { formação óssea. (fins regenerativos). }\end{array}$ \\
\hline M. López-Álvarez; et.al & $\begin{array}{l}\text { Revestimento de hidroxiapatita com sílica em células } \\
\text { semelhantes a osteoblastos. (fins regenerativos). }\end{array}$ \\
\hline Xiaoli Lu; et.al & $\begin{array}{l}\text { Material componente de cerâmicas, garantindo um bom } \\
\text { desempenho clínico. }\end{array}$ \\
\hline Jeong-Hun Choi; et.al & $\begin{array}{l}\text { Componente de um material de moldagem, sendo capaz } \\
\text { de reduzir o tempo de presa, porcentagem de } \\
\text { deformação máxima do molde e aumentar a } \\
\text { viscosidade e a resistência à tração. }\end{array}$ \\
\hline
\end{tabular}

Fonte: Autores (2021).

Dentre os estudos analisados, dois analisaram o uso de terras diatomáceas para fins regenerativos em tecidos ósseos; um a relevância da presença de diatomita em cerâmicas e, por fim, o último avaliou propriedades físicas de diferentes variações de um material odontológico para moldagem que possui diatomita em sua composição (Wang et al., 2014) (LópezÁlvarez et al., 2009) (Lu et al., 2012) (Choi et al., 2011).

\section{Discussão}

Devido ao baixo número de estudos encontrados, pode-se notar que o uso da terra de diatomáceas na composição de materiais odontológicos é pouco pesquisado e debatido, principalmente, no que se diz respeito a pesquisas não laboratoriais. Porém, os estudos inclusos discorrem de maneira ampla e permitem uma visão do uso da diatomita.

Dois dos estudos selecionados apresentam a diatomita e suas possibilidades na regeneração de tecidos ósseos, tornando esse material uma possível solução para os defeitos ósseos maxilares a mandibulares que são uns dos principais desafios na aplicação de implantes dentários (Koga et al., 2016).

O estudo de Wang et.al (Wang et al., 2014) destaca as possibilidades do uso das enzimas biosílica e biocalcita como catalisadores da formação de sílica e, consequentemente, reparadores de tecido. Os autores destacam que a biosílica é um biomaterial novo que apresenta um futuro brilhante na área da medicina regenerativa. Além disso, ressalta-se que o seu uso se 
torna possível devido ao acúmulo de biossílica nos tecidos adjacentes as áreas de formação óssea no organismo humano (R. Jugdaohsingh, 2007). Sendo assim, a sílica como material e a biosílica como biomaterial catalisador da reação química seriam capazes de induzir a regeneração óssea, por meio do efeito benéfico nos osteoblastos e efeitos adversos nos osteoclastos (Wiens et al., 2010) (Schröder et al., 2012).

A terra de diatomácea também teve seu uso como material bioregenerativo investigado em um estudo laboratorial que objetivou avaliar a influência de revestimentos de hidroxiapatita substituída por silício sobre uma célula de linhagem semelhante ao osteoblasto humano. Foram utilizadas duas diferentes linhagens de silício, uma de origem natural proveniente da terra de diatomáceas e outra de origem sintética. Os efeitos dos revestimentos de hidroxiapatita modificados foram avaliados sob os critérios de adesão e a proliferação das células semelhantes a osteoblastos. (López-Álvarez et al., 2009)

Por meio desta pesquisa comprovou-se que os dois tipos de revestimentos não acarretaram nenhum sinal de citotoxicidade. Além disso, o revestimento que possuía silício de origem natural apresentou, após 7 dias, com diferença significativa, melhores dados de proliferação e atividade celular em comparação com os revestimentos utilizando sílica sintética, o que pode ser justificado pela presença de elementos minoritários na composição do revestimento (Solla et al., 2007).

Ademais, a terra de diatomáceas também foi pesquisada em um estudo laboratorial para analisar o desempenho de cerâmicas dentárias de zircônia com a diatomita em sua composição. Foram testadas cerâmicas apresentando diferentes composições, mas todas com diatomita sendo aplicada da mesma maneira, por meio da técnica layer by layer, uma aplicação de camadas (Lu et al., 2012)

Após os diferentes testes para determinar as propriedades mecânicas das cerâmicas modificadas com diatomita, conclui-se que a técnica layer by layer foi capaz de garantir uma distribuição mais satisfatória das partículas de diatomita, além disso, as propriedades das cerâmicas modificadas foram melhoradas significativamente. Por exemplo, a diatomita garantiu uma maior resistência ao cisalhamento quando houve comparação entre as cerâmicas modificadas e as cerâmicas puras (Lu et al., 2012) Algumas das razões para a melhora das prioridades físicas das cerâmicas seriam a distribuição de tamanho das partículas e menor aglomeração, o que permitiriam uma resistência mecânica similar às cerâmicas convencionais (Guazzato et al., 2004b, 2004a).

Assim como nas cerâmicas, a terra de diatomáceas também pode ser usada para melhora das propriedades em materiais de moldagem. Como foi observado por Choi et. al (Choi et al., 2011), por meio de um estudo que comparou as propriedades físicas do material polivinilsiloxano (PVS) em diferentes combinações de sílica nanomérica. Os autores constataram que a combinação com terra de diatomáceas foi capaz de reduzir o tempo de presa (aumentando o tempo de trabalho); diminuir a porcentagem máxima de deformação (garantindo estabilidade da moldagem); aumento da viscosidade (facilitando a cópia de cada detalhe da arcada dentária) e resistência à tração (Choi et al., 2011)

É perceptível, que todas as pesquisas encontradas tratavam-se de estudos laboratoriais, os quais apresentam menor impacto científico quando comparados a estudos com seres humanos. Todavia, esses trabalhos, mesmo assim, apresentam valor científico significativo, pois servem como base para estudos futuros que poderão ser realizados.

Por fim, a limitação principal deste estudo é o fato de não ser uma revisão sistemática, tipo de estudo que apresenta critérios de avaliação e seleção mais rigorosos quando comparados a uma revisão integrativa. Contudo, essa limitação não desmerece a relevância deste trabalho, pois ele respondeu sua pergunta norteadora e foi capaz de expor o potencial notório de desenvolvimento e aplicação da diatomita como um material a ser pesquisado em diferentes estudos in vitro ou in vivo e, posteriormente, aplicado em diferentes materiais odontológicos. 


\section{Conclusão}

Diante do exposto pode-se concluir que a terra de diatomáceas pode ser utilizada em diferentes tipos de materiais odontológicos, principalmente, com o objetivo de garantir melhores propriedades físicas e mecânicas. Além disso, foi observado o baixo número de trabalhos publicados sobre esse tema, dessa forma torna-se necessário a realização de novas pesquisas, preferencialmente ensaios clínicos com materiais restauradores, de moldagem e de fins bioregenerativos com terra de diatomáceas em sua composição, para analisar por completo as possibilidades de uso da diatomita.

\section{Agradecimentos}

O presente estudo foi realizado com o apoio da Coordenação de Aperfeiçoamento de Pessoal de Nível Superior Brasil (CAPES) - Código de Financiamento 001.

\section{Referências}

Chain, M. (2013). Materiais Dentários (Série ABENO). Artes Médicas.

Choi, J. H., Kim, M. K., Woo, H. G., Song, H. J., \& Park, Y. J. (2011). Modulation of physical properties of polyvinylsiloxane impression materials by filler type combination. Journal of Nanoscience and Nanotechnology, 11(2), 1547-1550. https://doi.org/10.1166/jnn.2011.3332

Ediz, N., Bentli, İ., \& Tatar, İ. (2010). Improvement in filtration characteristics of diatomite by calcination. International Journal of Mineral Processing, 94(34). https://doi.org/10.1016/j.minpro.2010.02.004

Elias, Z., Poirot, O., Fenoglio, I., Ghiazza, M., Danière, M., Terzetti, F., Darne, C., Coulais, C., Matekovits, I., \& Fubini, B. (2006). Surface Reactivity, Cytotoxic, and Morphological Transforming Effects of Diatomaceous Earth Products in Syrian Hamster Embryo Cells. Toxicological Sciences, 91(2). https://doi.org/10.1093/toxsci/kfj177

Guazzato, M., Albakry, M., Ringer, S. P., \& Swain, M. v. (2004a). Strength, fracture toughness and microstructure of a selection of all-ceramic materials. Part I. Pressable and alumina glass-infiltrated ceramics. Dental Materials, 20(5). https://doi.org/10.1016/j.dental.2003.05.003

Guazzato, M., Albakry, M., Ringer, S. P., \& Swain, M. v. (2004b). Strength, fracture toughness and microstructure of a selection of all-ceramic materials. Part II. Zirconia-based dental ceramics. Dental Materials, 20(5). https://doi.org/10.1016/j.dental.2003.05.002

Guiry, M. (1997). Book reviews. European Journal of Phycology, 32(2). https://doi.org/10.1080/09670269710001737139

Hadjadj-Aoul, O., Belabbes, R., Belkadi, M., \& Guermouche, M. H. (2005). Characterization and performances of an Algerian diatomite-based gas chromatography support. Applied Surface Science, 240(1-4). https://doi.org/10.1016/j.apsusc.2004.06.108

Holmes, S. M., Graniel-Garcia, B. E., Foran, P., Hill, P., Roberts, E. P. L., Sakakini, B. H., \& Newton, J. M. (2006). A novel porous carbon based on diatomaceous earth. Chemical Communications, 25. https://doi.org/10.1039/b600708b

Koga, T., Minamizato, T., Kawai, Y., Miura, K., I, T., Nakatani, Y., Sumita, Y., \& Asahina, I. (2016). Bone Regeneration Using Dentin Matrix Depends on the Degree of Demineralization and Particle Size. PLOS ONE, 11(1). https://doi.org/10.1371/journal.pone.0147235

López-Álvarez, M., Solla, E. L., González, P., Serra, J., León, B., Marques, A. P., \& Reis, R. L. (2009). Silicon-hydroxyapatite bioactive coatings (Si-HA) from diatomaceous earth and silica. Study of adhesion and proliferation of osteoblast-like cells. Journal of Materials Science: Materials in Medicine, 20(5), 1131-1136. https://doi.org/10.1007/s10856-008-3658-0

Losic, D., Mitchell, J. G., \& Voelcker, N. H. (2009). Diatomaceous Lessons in Nanotechnology and Advanced Materials. Advanced Materials, 21(29). https://doi.org/10.1002/adma.200803778

Lu, X., Xia, Y., Liu, M., Qian, Y., Zhou, X., Gu, N., \& Zhang, F. (2012). Improved performance of diatomite-based dental nanocomposite ceramics using layer-by-layer assembly. International Journal of Nanomedicine, 7, 2153-2164. https://doi.org/10.2147/IJN.S29851

Ouzzani, M., Hammady, H., Fedorowicz, Z., \& Elmagarmid, A. (2016). Rayyan —a web and mobile app for systematic reviews. Systematic Reviews, 5(1). https://doi.org/10.1186/s13643-016-0384-4

R. Jugdaohsingh. (2007). Silicon And Bone Health. J Nutr Health Aging, 11(2), 99-110.

Şan, O., Gören, R., \& Özgür, C. (2009). Purification of diatomite powder by acid leaching for use in fabrication of porous ceramics. International Journal of Mineral Processing, 93(1). https://doi.org/10.1016/j.minpro.2009.04.007

Schröder, H. C., Wang, X. H., Wiens, M., Diehl-Seifert, B., Kropf, K., Schloßmacher, U., \& Müller, W. E. G. (2012). Silicate modulates the cross-talk between osteoblasts (SaOS-2) and osteoclasts (RAW 264.7 cells): Inhibition of osteoclast growth and differentiation. Journal of Cellular Biochemistry, 113(10). https://doi.org/10.1002/jcb.24196

Solla, E. L., Borrajo, J. P., González, P., Serra, J., Chiussi, S., León, B., \& López, J. G. (2007). Study of the composition transfer in the pulsed laser deposition of silicon substituted hydroxyapatite thin films. Applied Surface Science, 253(19). https://doi.org/10.1016/j.apsusc.2007.02.116 
Research, Society and Development, v. 10, n. 16, e521101623846, 2021

(CC BY 4.0) | ISSN 2525-3409 | DOI: http://dx.doi.org/10.33448/rsd-v10i16.23846

Souza, M. T. de, Silva, M. D. da, \& Carvalho, R. de. (2010). Integrative review: what is it? How to do it? Einstein (São Paulo), 8(1). https://doi.org/10.1590/s1679-45082010rw1134

Wang, X., Schröder, H. C., \& Müller, W. E. G. (2014). Enzyme-based biosilica and biocalcite: Biomaterials for the future in regenerative medicine. In Trends in Biotechnology 32(9), 441-447. Elsevier Ltd. https://doi.org/10.1016/j.tibtech.2014.05.004

Wiens, M., Wang, X., Schröder, H. C., Kolb, U., Schloßmacher, U., Ushijima, H., \& Müller, W. E. G. (2010). The role of biosilica in the osteoprotegerin/RANKL ratio in human osteoblast-like cells. Biomaterials, 31(30). https://doi.org/10.1016/j.biomaterials.2010.07.002 